\title{
Structural instability of two- and three-dimensional pyrochlore spin lattices in high magnetic fields
}

\author{
Oleg Derzhko ${ }^{1,2,3}$ and Johannes Richter ${ }^{2}$ \\ ${ }^{1}$ Institute for Condensed Matter Physics, National Academy of Sciences of Ukraine, \\ 1 Svientsitskii Street, L'viv-11, 79011, Ukraine \\ ${ }^{2}$ Institut für Theoretische Physik, Universität Magdeburg, \\ P.O. Box 4120, D-39016 Magdeburg, Germany \\ ${ }^{3}$ National University "Lvivska Politechnika", \\ 12 S. Bandera Street, L'viv, 79013, Ukraine
}

August 22, 2018

\begin{abstract}
We consider the quantum spin- $s X X Z$ Heisenberg antiferromagnet on the two- and three-dimensional pyrochlore lattices and examine a spin-Peierls mechanism of lowering the total energy by a lattice distortion in a high magnetic field. For the exact eigenstates consisting of several independent localized magnons in a ferromagnetic environment we show the existence of a spin-Peierls instability by rigorous analytical calculations. In addition we report on exact diagonalization data for finite two-dimensional pyrochlore lattices up to $N=64$ sites. We discuss a scenario of the field-tuned changes in the groundstate properties of the frustrated spin lattices due to the coupling between spin and lattice degrees of freedom.
\end{abstract}

PACS number(s): $75.10 . \mathrm{Jm}, 75.45 .+\mathrm{j}$

Keywords: checkerboard spin lattice, pyrochlore spin lattice, high magnetic fields, spin-Peierls instability

\section{Introduction. Independent localized-magnon states}

Antiferromagnetically interacting Heisenberg spins on geometrically frustrated lattices have attracted much attention of physicists during last years. Frustrating interactions may lead to a rich phase diagram both on the classical and quantum levels [1, 2, 3, 4. In the presence of an external magnetic field frustrated spin systems exhibit a number of specific properties. In particular, plateaus and jumps can be observed in the zero-temperature magnetization curve for such models. Another intriguing property is a magnetic-field induced spin-Peierls effect in frustrated systems to be discussed below.

A particular case of geometrically frustrated lattices is the pyrochlore lattice (i.e. a network of cornersharing tetrahedra) which is especially attractive since it has many experimental realizations. The twodimensional (2D) version of the pyrochlore network, also known as the planar pyrochlore, the checkerboard lattice, or the square lattice with crossings, has the same local coordination as in the three-dimensional (3D) case, however, it is easier for numerical studies. The studies of this simpler model can be helpful in understanding of its 3D analog [5, 6, 7, 8,

There is a number of experimental and theoretical papers discussing the effects of magnetoelastic couplings in geometrically frustrated antiferromagnets. Because of frustration, such systems can have a huge degeneracy of the ground state which prevents the system from finding a unique ground state as temperature 
decreases. Any perturbation can have a dramatic effect selecting one ground state over another. Different perturbations which can lift the ground-state degeneracy have been discussed in the literature, and a magnetoelastic coupling is among them. This coupling allows the spin lattice to distort thus relieving the magnetic frustration and lowering the total energy of the system. Much attention in recent years has been paid to such spin-Peierls-like phase transitions in the pyrochlore antiferromagnets from the experimental as well as from the theoretical side. Thus, inelastic magnetic neutron scattering on the 3D pyrochlore antiferromagnet $\mathrm{ZnCr}_{2} \mathrm{O}_{4}$ (the spin-3/2 $\mathrm{Cr}^{3+}$ ions form the 3D pyrochlore lattice) revealed that a lattice distortion can lower the energy driving the spin system into an ordered phase [9]. Further probes of a lattice distortion in $\mathrm{ZnCr}_{2} \mathrm{O}_{4}$ using neutron scattering and infrared spectroscopy have been reported in Refs. 10, 11. NMR investigation of $\mathrm{Y}_{2} \mathrm{Mo}_{2} \mathrm{O}_{7}$ (the spin-1 $\mathrm{Mo}^{4+}$ ions form the 3D pyrochlore lattice) gives evidence for discrete lattice distortions which reduce the energy of the system [12. The results of a similar $\mu \mathrm{SR}$ study for this compound have been reported recently in Ref. [13]. A lifting of a macroscopic ground-state degeneracy of frustrated magnets through a coupling between spin and lattice degrees of freedom in pyrochlore antiferromagnets was studied theoretically in Refs. [14, 15, 16] and the relation of these theoretical studies to pyrochlore compounds was discussed. More recently, inspired from the experimental data on $\mathrm{ZnCr}_{2} \mathrm{O}_{4}$ [10] the authors of Ref. [17] have examined a model of lattice-coupled antiferromagnetic classical spins on the pyrochlore lattice. They conclude that a particular pattern - a hexagon contraction - may arise owing to a lattice distortion which reduces the energy. While the above mentioned studies refer to zero-field case, Penc and coworkers [18, 19] have considered the magnetoelastic coupling at finite magnetic fields and have shown that the coupling between classical spin and lattice degrees of freedom in a pyrochlore antiferromagnet stabilizes a half-magnetization plateau which occurs at intermediate fields. This conclusion agrees with the high-field measurements at up to $47 \mathrm{~T}$ for the $s=3 / 2$ Heisenberg pyrochlore antiferromagnet $\mathrm{CdCr}_{2} \mathrm{O}_{4}$ [20].

In what follows, we want to discuss the impact of localized-magnon states [21, 22, 23] on the stability of the $2 \mathrm{D}$ and $3 \mathrm{D}$ pyrochlore lattices against distortions. In contrast to the above mentioned zero-field studies on the effects of magnetoelastic couplings in the pyrochlore lattice, high magnetic fields are essential in our consideration since only in a high magnetic field the independent localized magnons may become relevant for the ground-state properties of the considered systems. The present study extends our earlier results referring to the square-kagomé and kagomé lattices [24, 25].

To be specific, we consider two geometrically frustrated lattices, namely, the 2D pyrochlore lattice (Figs. 112) and the 3D pyrochlore lattice (Figs. 3 4). The Hamiltonian of $N$ quantum spins of length $s$ reads

$$
H=\sum_{(n m)} J_{n m}\left(\frac{1}{2}\left(s_{n}^{+} s_{m}^{-}+s_{n}^{-} s_{m}^{+}\right)+\Delta s_{n}^{z} s_{m}^{z}\right)-h S^{z}
$$

Here the sum runs over the bonds (edges) which connect the neighboring sites (vertices) on the spin lattice under consideration, $J_{n m}>0$ are the antiferromagnetic exchange constants between the sites $n$ and $m$, $\Delta \geq 0$ is the exchange interaction anisotropy parameter, $h$ is the external magnetic field, and $S^{z}=\sum_{n} s_{n}^{z}$ is the $z$-component of the total spin. We assume that all bonds in the lattice without distortion have the same length and hence all exchange constants have the same value $J$.

We begin with a brief illustration of the independent localized-magnon states in the considered systems (the localized magnons in the uniform 2D and 3D pyrochlore lattices were also considered in Refs. [23] and [22], respectively; for a review on localized magnons see Refs. [26, 27]). The localized-magnon states are excitations above the fully polarized ferromagnetic state, which is the ground state of the antiferromagnet in magnetic fields exceeding the saturation field $h_{1}$. The localized magnons live on restricted areas of the 


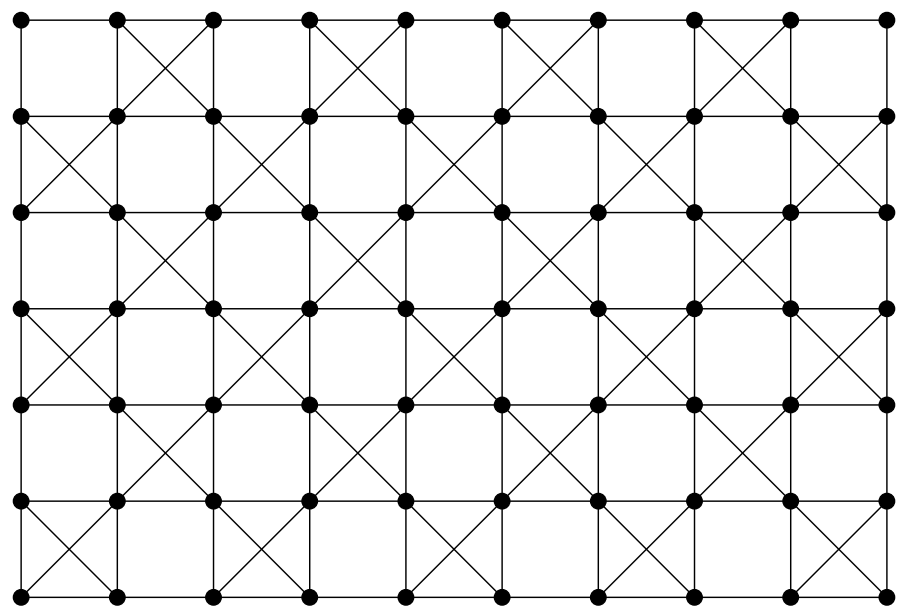

Figure 1: The undistorted two-dimensional pyrochlore lattice. All bonds have the same strength $J$. In what follows we use as shorthands the notations "empty squares" (squares without bond-crossings) and "crossed squares" (squares with bond-crossings which are, in fact, projected tetrahedra).

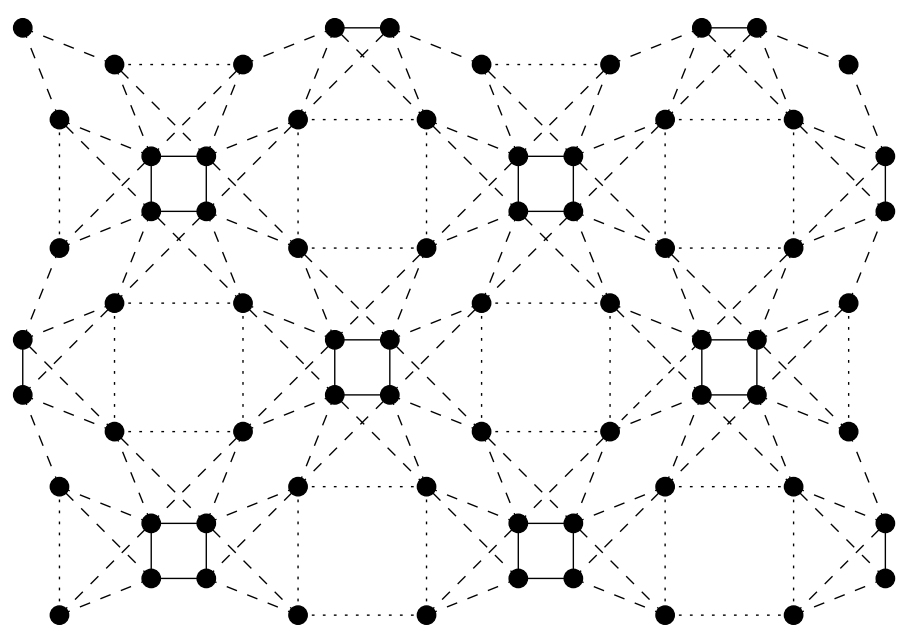

Figure 2: The distorted two-dimensional pyrochlore lattice. The solid and the dotted bonds have the strengths $J(1+\sqrt{2} \delta)$ and $J(1-\sqrt{2} \delta)$, respectively, the dashed bonds have the strength $J$ (with an accuracy up to $\delta$ ). Note that the lattice volume remains unchanged after such a distortion. 


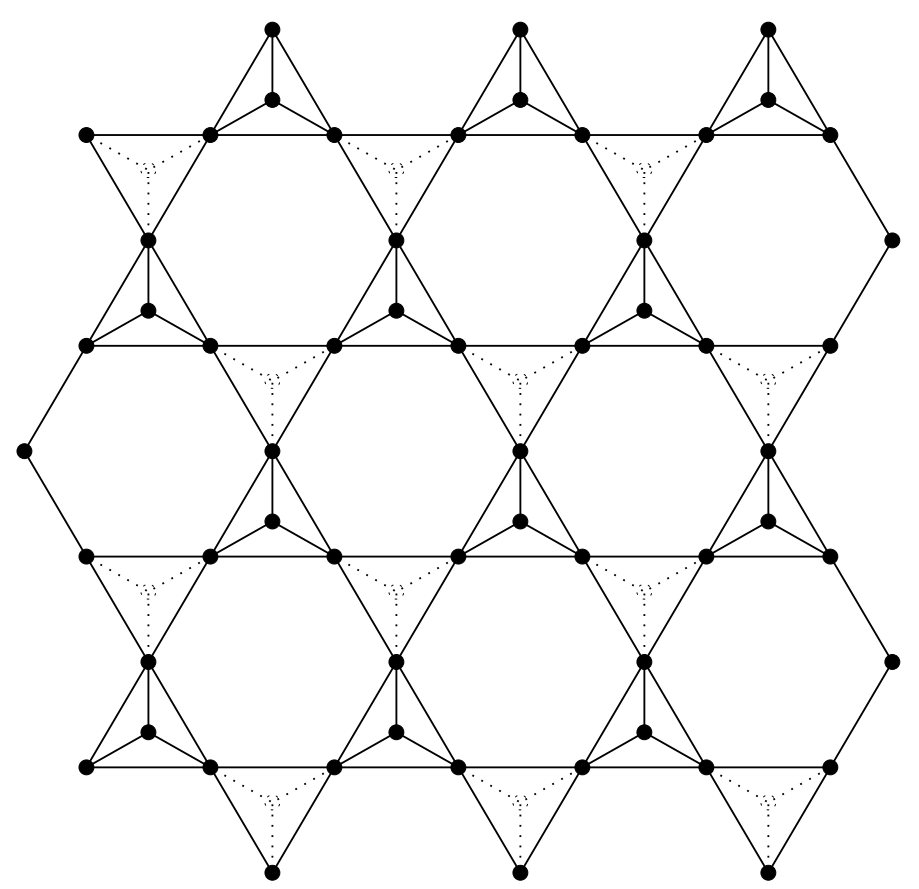

Figure 3: The $\{111\}$ slice of the undistorted three-dimensional pyrochlore lattice. All bonds have the same strength $J$.

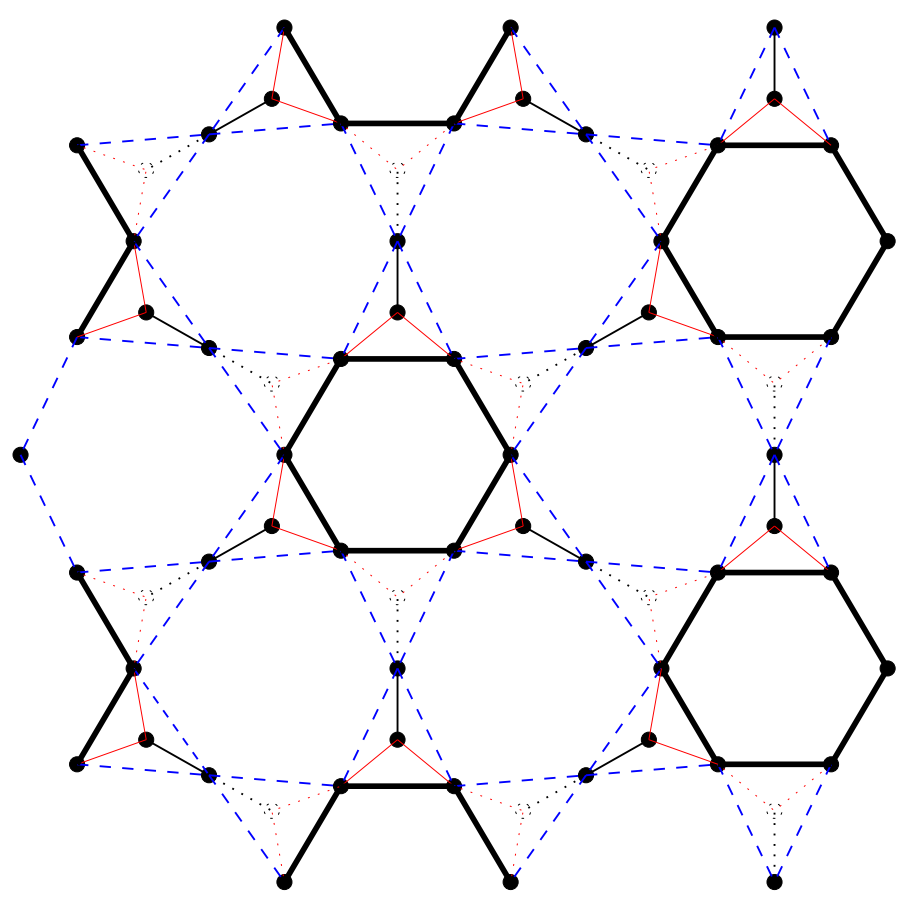

Figure 4: (color online). The distorted $\{111\}$ slice of the three-dimensional pyrochlore lattice. The thick solid bonds (thick black) represent the shrinked hexagons and have the strength $J(1+\delta)$, the thin solid and dotted bonds (red) have the strength $J(1-\delta /(2 \sqrt{3}))$, the dashed bonds (blue) have the strength $J(1-\delta / 2)$. The normal solid and dotted bonds (normal black) remain as in the undistorted lattice having the strength $J$. Note that the lattice volume remains unchanged after such a distortion. 
lattice, e.g. on the empty squares of the $2 \mathrm{D}$ pyrochlore lattice or on the hexagons of 3D pyrochlore lattice. They represent exact eigenstates of the frustrated spin lattice and become ground states in high magnetic fields.

In the $2 \mathrm{D}$ pyrochlore lattice without distortion the energy of the eigenstate with one magnon localized on an empty square is

$$
E_{1}=-2 s J+2 s(2 s-1) J \Delta+4 s(4 s-1) J \Delta+(3 N-20) s^{2} J \Delta .
$$

Here the first and the second terms represent the contribution from the bonds along the square, the third term represents the contribution from the bonds which connect the square with the environment, and the fourth term represents the contribution from the ferromagnetic environment. For $n$ independent localized magnons we have instead of (2)

$$
E_{n}=3 N s^{2} J \Delta-n \epsilon_{1}, \quad \epsilon_{1}=2 s J(1+3 \Delta)
$$

and $n$ may vary from 1 to $n_{\max }=N / 8$ [23. The maximal value of the filling number $n_{\max }$ corresponds to a dense magnon crystal in which magnons sit on every forth empty square. This magnon crystal state is four-fold degenerate and it breaks spontaneously the translational symmetry of the lattice.

Let us pass to the $3 \mathrm{D}$ pyrochlore lattice without distortions. This lattice can be composed from alternating kagomé-like and triangular planes stacked along the [111] direction. The localized magnons can exist in a kagomé-like plane (i.e. the $\{111\}$ slice of the 3D pyrochlore lattice considered in Ref. [28]) and are trapped on hexagons. The $\{111\}$ slice of the undistorted 3D pyrochlore lattice is shown in Fig. 3. The eigenstate with one magnon trapped on a hexagon has the energy given by Eq. (2) (however, with the following contributions of different bonds: $-2 s J+2 s(3 s-1) J \Delta$ (along the edges of the hexagon), $4 s(6 s-1) J \Delta$ (along the edges connecting the hexagon with the environment), $(3 N-30) s^{2} J \Delta$ (ferromagnetic environment)). For $n$ independent localized magnons we again arrive at Eq. (3), however, $n$ may vary from 1 to $n_{\max }=N / 12$ [22]. The magnon crystal state corresponds to maximum filling $n_{\max }$. In this magnon crystal state every third hexagon in the $\{111\}$ slice is occupied by magnon producing thus three-fold degeneracy within a slice (as for the kagomé lattice). Since these three different crystal states can be chosen independently in each slice, the degeneracy of the magnon crystal of the whole 3D pyrochlore lattice is much higher and scales at least as $3^{L}$ where $L \sim N^{1 / 3}$ is the number of the $\{111\}$ slices in the $3 \mathrm{D}$ pyrochlore lattice.

Each magnon decreases $S^{z}$ by 1 and the localized-magnon state with $n$ independent localized magnons has $S^{z}=N s-n$. These states have the lowest energies in the corresponding sectors of $S^{z}$ [21, 29]. Hence, Eq. (3) with $n=N s-S^{z}$ gives $E_{\min }\left(S^{z}\right)$ for the $2 \mathrm{D}$ and 3D pyrochlore antiferromagnets when $S^{z}=N s, N s-1, \ldots, N s-n_{\max }$.

In the presence of an external field we must add to the energy (3) the Zeeman term $-h S^{z}=-h(N s-n)$. Then the ground state belongs to an $S^{z}$ determined by the condition $h=\partial E\left(S^{z}\right) / \partial S^{z}$. At the saturation field $h_{1}=\epsilon_{1}$ the energy of all localized-magnon states with $n=1, \ldots, n_{\max }$ is the same and the zero-temperature magnetization $S^{z}$ jumps between the saturation value $N s$ and the value $N s-n_{\max }$.

On the basis of general arguments [30, 31] (see also Refs. 44, 24, 27]) we expect that the magnon crystal state has gapped excitations and hence the system exhibits a magnetization plateau at $S^{z}=N s-n_{\max }$ between $h_{2}=h_{1}-\Delta h$ and $h_{1}$. Numerical data support this expectation. Thus, a finite-size analysis of the data for the plateau width obtained for the spin-1/2 isotropic Heisenberg antiferromagnet on finite 2D pyrochlore lattices with $N=24,32,40,48,64$ yields for $\Delta h$ about $0.2 J$. Since we know e.g. from 
the triangular lattice [32, 33] and the square-kagomé and kagomé lattices [24, 25], that the plateau width depends on the spin quantum number $s$ we have also calculated the plateau width $\Delta h$ for the spin-s isotropic Heisenberg system on the 2D pyrochlore lattice with $N=24$ sites for the values of $s=1 / 2,1,3 / 2,2,5 / 2$. We find that $\Delta h$ scales excellently as $\sqrt{s}$ leading to a relative plateau width $\Delta h / h_{1} \sim 1 / \sqrt{s}$ vanishing in the classical limit $s \rightarrow \infty$.

Finally, we want to underline that the ground state of both pyrochlore quantum antiferromagnets exhibits a huge degeneracy at the saturation field owing to the localized-magnon states. This happens because a certain trapping cell of the lattice can be either occupied by a magnon or not whereas the energies of the states with different number of magnons are the same. As a result, the degeneracy $\mathcal{W}$, that is the number of ways to place magnons on the lattice, grows exponentially with $N$ with a crude estimate $\mathcal{W}>2^{n_{\max }}$ [4] This estimate of the ground-state degeneracy can be improved after mapping the lattice with localized magnons onto an auxiliary lattice occupied by hard-core objects [34, 35, 36, 27].

\section{Lattice instability in high magnetic fields}

We want to examine the lattice stability with respect to a spin-Peierls mechanism in high magnetic fields. For that we consider a small lattice deformation which does not violate the conditions for the existence of localized-magnon ground states, and analyze the change of the total energy which consists of the magnetic and the elastic parts. We search for a maximal gain of the magnetic energy due to lattice deformation. For the magnon crystal state the Eqs. (2), (3) give a hint how such a deformation may be constructed. Actually, for the magnon crystal state the nearest-neighbor spin correlation functions are distributed inhomogeneously. Along the empty square or hexagon hosting a localized magnon the transverse correlations $\left\langle s_{i}^{x} s_{i+1}^{x}\right\rangle+\left\langle s_{i}^{y} s_{i+1}^{y}\right\rangle$ are negative, but all other nearest-neighbor correlations are positive. Therefore, one may expect a maximal gain of magnetic energy by increasing the (antiferromagnetic) bonds on the empty square or hexagon which hosts localized magnon and decreasing the (antiferromagnetic) bonds on the attaching triangles. The corresponding deformations for the 2D and 3D pyrochlore lattices are discussed below. The changes of the magnetic energy due to such deformations are calculated analytically for the values of $S^{z}$ corresponding to the magnon crystal. Furthermore we present numerical data for lower values of $S^{z}$ in the $2 \mathrm{D}$ case.

\subsection{Two-dimensional pyrochlore spin lattice}

A pattern of distortions which uses the inhomogeneous distribution of nearest-neighbor spin correlations in an optimal way and does not violate the conditions of existence of the magnon crystal state is shown in Fig. 2 This lattice deformation corresponds to the $T_{2}$ vibration mode of isolated tetrahedron (see Ref. [16]), i.e. in every tetrahedron a pair of opposite to each other bonds (belonging to empty squares in Fig. (1) are stretched and contracted by the same amount. Introducing the parameter $\delta$ which is proportional to the displacement of the sites we find the following changes of the exchange interactions (with an accuracy up to linear terms in $\delta$ )

$$
J \rightarrow J(1 \pm \sqrt{2} \delta), \quad J \rightarrow J
$$

The stronger (weaker) bonds are shown in Fig. 2 by solid (dotted) lines; the bonds having unchanged strength are shown in Fig. 2 by dashed lines. As a result, the magnetic energy of the distorted magnon 
crystal state

$$
E_{n_{\max }}(\delta)=3 N s^{2} J \Delta-n_{\max }\left(\epsilon_{1}+2 \sqrt{2} s(1+\Delta) J \delta\right)
$$

decreases proportionally to $\delta$. The deformation of the 24 bonds forming one cell belonging to a localizedmagnon area increases the elastic energy by $8 \gamma(\sqrt{2} \delta)^{2}$ where the parameter $\gamma$ is proportional to the elastic constant of the lattice. As a result, for the distorted magnon crystal state the elastic energy $16 \gamma \delta^{2} n_{\max }$ increases proportionally to $\delta^{2}$. Combining the magnetic and the elastic energies of the deformed lattices we find that the total energy for the magnon crystal state has a minimum at $\delta=\delta^{\star}=(\sqrt{2} / 16) s(1+\Delta) J / \gamma \neq 0$ that explicitly demonstrates the lattice instability.

So far our results are rigorous. Now the question arises whether the lattice distortion shown in Fig. 2 remains energetically favorable for $S^{z}<N s-n_{\max }$. To discuss this question we perform exact diagonalization for finite lattices using J. Schulenburg's spinpack [37]. Assuming that the lowest magnetic energy for a given value of $S^{z}$ depends on $\delta$ like $E_{\min }(\delta)=E_{\min }(0)+A \delta^{p}$ and taking $\delta$ of the order $10^{-5} \ldots 10^{-4}$ we estimate the exponent $p$ numerically. If $p$ is less than 2 (and $A<0$ ) the lattice deformation for the considered finite lattice is favorable, otherwise it is not. We have calculated the exponent $p$ for the $s=1 / 2$ isotropic $(\Delta=1)$ Heisenberg system on finite lattices of $N=32,40$ and 48 sites in the two sectors of $S^{z}$ just below the sector of the magnon crystal state, i.e. for $S^{z}=N s-n_{\max }-1$ and $S^{z}=N s-n_{\max }-2$. Note that for $N=48$ with $S^{z}=N s-n_{\max }-2=16$ the lowest eigenvalues of a Hamiltonian matrix of size $6 \cdot 10^{7} \times 6 \cdot 10^{7}$ must be calculated. We obtain a gain in magnetic energy (i.e. $A<0$ ) in all cases. In the sector $S^{z}=\frac{1}{2} N-n_{\max }-1$ we obtain $p=1.0,2.0,2.0$ for $N=32,40,48$, respectively. The "outrider" for $N=32$ may be attributed to finite-size effects, indeed we find $p=2.0$ in the next sectors below $S^{z}=\frac{1}{2} N-n_{\max }-1$. For $N=40$ and $N=48$ the exponent remains $p=2.0$ in the sector $S^{z}=\frac{1}{2} N-n_{\max }-2$.

Thus, our analytical calculations demonstrate a spin-Peierls lattice instability of the 2D pyrochlore antiferromagnet for the lowest energy states in the sectors of $S^{z}=N s-n_{\max }, \ldots, N s-1$. The lattice deformation is not favorable in the fully polarized state when $S^{z}=N s$. Our numerical calculations give evidence that it also disappears when $S^{z}<N s-n_{\max }$.

Our findings imply that the 2D pyrochlore quantum antiferromagnet at zero temperature being placed in a high magnetic field $h>h_{1}$ becomes distorted when the applied field is lowered to the saturation field $h_{1}=\epsilon_{1}$, and the lattice deformation disappears when the field becomes smaller than the field at the left endpoint of the plateau $h_{2}$. It should be noted that a hysteresis phenomenon in the vicinity of the saturation field is expected: Starting from a large magnetic field $h>h_{1}$ and then decreasing the field the distortion sets in at $h_{1}=\epsilon_{1}$. On the other hand, starting from a field below the saturation field and then increasing the field the distorted magnon crystal state survives until the saturation field of the distorted lattice $h_{1}\left(\delta^{\star}\right)=\epsilon_{1}+(1 / 8) s^{2}(1+\Delta)^{2} J^{2} / \gamma$.

\subsection{Three-dimensional pyrochlore spin lattice}

We proceed along the same lines switching to the 3D pyrochlore lattice. A lattice distortion which does not violate the conditions for the existence of localized magnons may be constructed by appropriate deformations within the kagomé planes of the $\{111\}$ slices. Such a pattern of distortions is illustrated in Fig. 4 Note that the triangular planes and the distance between the triangular and the kagomé planes remain unchanged. The shrinked hexagons form a $\sqrt{3} \times \sqrt{3}$ structure, all other sites of the $\{111\}$ slice not belonging to shrinked hexagons do not change their positions (compare Figs. 3 and 4). The described deformation is similar but 
not identical to the one considered in Ref. [17. The deformation discussed in Ref. [17] assumes that each hexagon contracts towards its center of mass and this distortion does not support the localized magnons. Denoting by $\delta$ the parameter which is proportional to the displacement of the mentioned sites we arrive at the following change of the exchange interactions (with an accuracy up to linear terms in $\delta$ )

$$
J \rightarrow J(1+\delta), \quad J \rightarrow J\left(1-\frac{1}{2 \sqrt{3}} \delta\right), \quad J \rightarrow J\left(1-\frac{1}{2} \delta\right)
$$

along the edges of the contracted hexagons (thick black lines in Fig. 4), along the out-of-plane triangles attached to the contracted hexagons (thin solid and dotted red lines in Fig. 四), along the in-plane triangles attached to the contracted hexagons (dashed blue lines in Fig. 4), respectively. The exchange interaction along the bonds which are not connected to the contracted hexagons (normal solid and dotted black lines in Fig. (4) remains unchanged and has the strength $J$. The magnetic energy of the distorted magnon crystal state then reads

$$
E_{n_{\max }}(\delta)=3 N s^{2} J \Delta-n_{\max }\left(\epsilon_{1}+s\left(2+\frac{6 s+\sqrt{3}-1}{\sqrt{3}} \Delta\right) J \delta\right),
$$

and the corresponding elastic energy is $10 \gamma \delta^{2} n_{\max }$. The total energy achieves a minimum at $\delta=\delta^{\star}=$ $(s / 20)(2+(6 s+\sqrt{3}-1) \Delta / \sqrt{3}) J / \gamma \neq 0$ demonstrating the lattice instability. Again this lattice deformation is accompanied by hysteresis phenomenon with the saturation field of the distorted lattice $h_{1}\left(\delta^{\star}\right)=\epsilon_{1}+$ $(1 / 40) s^{2}(2+(6 s+\sqrt{3}-1) \Delta / \sqrt{3})^{2} J^{2} / \gamma$.

Due to the higher dimensionality the finite-size effects for lattice sizes accessible with exact diagonalization are much more important for the 3D pyrochlore lattice and there are no reliable numerical data for the sectors below $S^{z}=N s-n_{\max }$. Therefore, the question about the stability of the lattice against the considered deformations for magnetic fields below the plateau belonging to the magnon crystal state remains open for the $3 \mathrm{D}$ case.

\section{Conclusions}

In the present paper we reported a spin-Peierls instability in high magnetic fields of the quantum spin- $s$ $X X Z$ Heisenberg antiferromagnet on the 2D and 3D pyrochlore lattices in adiabatic treatment. Since both lattices support localized-magnon states which are the lowest energy states in the corresponding sectors of $S^{z}$ an instability of the uniform lattice near the saturation field has been proved by rigorous analytical calculations. Results for smaller fields have been obtained by exact diagonalization. Although we cannot exclude that other distortions (different from the ones shown in Fig. 2 and Fig. 4) cannot come into the play, we have given arguments that the considered deformations are the most favorable ones taking advantage of localized magnons in an optimal way and we have shown explicitly that the undistorted lattice is unfavorable in the ground state with $S^{z}=N s-n_{\max }, \ldots, N s-1$.

It is interesting to note that while the lattice deformation for the $2 \mathrm{D}$ pyrochlore lattice (Fig. 22) occurs simultaneously over all lattice and hence lifts the degeneracy of the ground state at the saturation field, the

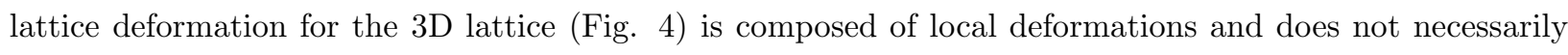
reduce the ground-state degeneracy at the saturation field.

From experimental point of view, it seems to be important to have a magnetization plateau where the deformation occurs, since that gives the possibility to observe the effect in a finite range of $h$. Observation of hysteresis in the magnetization and the deformation of pyrochlore lattices in the vicinity of the saturation field 
can be an experimental sign of the effect discussed theoretically in this paper. For experimental investigations of antiferromagnets near saturation field $h_{1}$ one needs materials with comparably small exchange interaction $J$ and small value of spin $s$ to be able to reach the high-field regime. Materials with small spin quantum number $s$ are more appropriate, also because the plateau near saturation vanishes with increasing $s$. We mention, that in our analysis we dealt with ideal pyrochlore geometry whereas in real compounds deviations may be expected. However, there are arguments that the effects of the localized magnons survive under slight deviations from the ideal geometry [34.

There are now several magnetic materials which can be modeled by the 3D pyrochlore antiferromagnet (for instance, spin- 1 systems such as $\mathrm{ZnV}_{2} \mathrm{O}_{4}, \mathrm{MgV}_{2} \mathrm{O}_{4}, \mathrm{Y}_{2} \mathrm{Mo}_{2} \mathrm{O}_{7}$, spin-3/2 systems such as $\mathrm{ZnCr}_{2} \mathrm{O}_{4}$, $\mathrm{CdCr}_{2} \mathrm{O}_{4}, \mathrm{HgCr}_{2} \mathrm{O}_{4}$; most of them with exchange constants of about $10^{2} \mathrm{~K}$ ) and there is some hope that certain features connected with the above presented scenario of a lattice instability driven by a magnetic field may be observed in antiferromagnetic compounds with pyrochlore structure.

Acknowledgments: The present study was supported by the DFG (project 436 UKR 17/17/03). O. D. acknowledges the kind hospitality of the Magdeburg University in the autumn of 2004 and in the summer of 2005. O. D. and J. R. are grateful to the MPIPKS Dresden for hospitality during the International Workshop on Collective quantum states in low-dimensional transition metal oxides (February 22 - 25, 2005). The authors thank A. Honecker, R. Moessner, K. Penc, and J. Schulenburg for discussions.

\section{References}

[1] R. Moessner, Can. J. Phys. 79, 1283 (2001).

[2] C. Lhuillier and G. Misguich, in "High Magnetic Fields: Applications in Condensed Matter Physics and Spectroscopy", C. Berthier, L. P. Levy and G. Martinez, Eds. (Lecture Notes in Physics, 595) (Springer, Berlin, 2002), pp.161-190; arXiv:cond-mat/0109146

[3] G. Misguich and C. Lhuillier, in "Frustrated Spin Systems", H. T. Diep, Ed. (World Scientific, Singapore, 2005), pp.229-306; arXiv:cond-mat/0310405

[4] J. Richter, J. Schulenburg, and A. Honecker, in "Quantum Magnetism", U. Schollwöck, J. Richter, D. J. J. Farnell and R. F. Bishop, Eds. (Lecture Notes in Physics, 645) (Springer, Berlin, 2004), pp.85-153.

[5] S. E. Palmer and J. T. Chalker, Phys. Rev. B 64, 094412 (2001).

[6] W. Brenig and A. Honecker, Phys. Rev. B 65, 140407(R) (2002).

[7] J. - B. Fouet, M. Mambrini, P. Sindzingre, and C. Lhuillier, Phys. Rev. B 67, 054411 (2003).

[8] O. Tchernyshyov, O. A. Starykh, R. Moessner, and A. G. Abanov, Phys. Rev. B 68, 144422 (2003).

[9] S. - H. Lee, C. Broholm, T. H. Kim, W. Ratcliff II, and S. - W. Cheong, Phys. Rev. Lett. 84, 3718 (2000).

[10] S. - H. Lee, C. Broholm, W. Ratcliff, G. Gasparovic, Q. Huang, T. H. Kim, and S. - W. Cheong, Nature 418, 856 (2002). 
[11] A. B. Sushkov, O. Tchernyshyov, W. Ratcliff II, S. W. Cheong, and H. D. Drew, Phys. Rev. Lett. 94, $137202(2005)$.

[12] A. Keren and J. S. Gardner, Phys. Rev. Lett. 87, 177201 (2001).

[13] E. Sagi, O. Ofer, A. Keren, and J. S. Gardner, Phys. Rev. Lett. 94, 237202 (2005).

[14] Y. Yamashita and K. Ueda, Phys. Rev. Lett. 85, 4960 (2000).

[15] O. Tchernyshyov, R. Moessner, and S. L. Sondhi, Phys. Rev. Lett. 88, 067203 (2002).

[16] O. Tchernyshyov, R. Moessner, and S. L. Sondhi, Phys. Rev. B 66, 064403 (2002).

[17] Chenglong Jia and Jung Hoon Han, arXiv:cond-mat/0412036 Chenglong Jia, Jung Ho Nam, June Seo Kim, and Jung Hoon Han, Phys. Rev. B 71, 212406 (2005).

[18] K. Penc, N. Shannon, and H. Shiba, Phys. Rev. Lett. 93, 197203 (2004).

[19] Y. Motome, K. Penc, and N. Shannon, arXiv:cond-mat/0506500

[20] H. Ueda, H. A. Katori, H. Mitamura, T. Goto, and H. Takagi, Phys. Rev. Lett. 94, 047202 (2005).

[21] J. Schnack, H. - J. Schmidt, J. Richter, and J. Schulenburg, Eur. Phys. J. B 24, 475 (2001).

[22] J. Schulenburg, A. Honecker, J. Schnack, J. Richter, and H. - J. Schmidt, Phys. Rev. Lett. 88, 167207 (2002).

[23] J. Richter, J. Schulenburg, A. Honecker, J. Schnack, and H. - J. Schmidt, J. Phys.: Condens. Matter 16, S779 (2004).

[24] J. Richter, O. Derzhko, and J. Schulenburg, Phys. Rev. Lett. 93, 107206 (2004).

[25] O. Derzhko, J. Richter, and J. Schulenburg, submitted to physica status solidi (b) (2005).

[26] J. Richter, Fizika Nizkikh Temperatur (Kharkiv) 31, 832 (2005) [Low Temperature Physics 31, ??? (2005)]; arXiv:cond-mat/0503243

[27] M. E. Zhitomirsky and H. Tsunetsugu, arXiv:cond-mat/0506327

[28] O. Tchernyshyov, Hong Yao, and R. Moessner, Phys. Rev. B 69, 212402 (2004).

[29] H. - J. Schmidt, J. Phys. A 35, 6545 (2002).

[30] T. Momoi and K. Totsuka, Phys. Rev. B 61, 3231 (2000).

[31] M. Oshikawa, Phys. Rev. Lett. 84, 1535 (2000).

[32] A. Honecker, J. Phys.: Condens. Matter 11, 4697 (1999); A. Honecker, J. Schulenburg, and J. Richter, J. Phys.: Condens. Matter 16, S749 (2004).

[33] A. V. Chubukov and D. I. Golosov, J. Phys.: Condens. Matter 3, 69 (1991).

[34] O. Derzhko and J. Richter, Phys. Rev. B 70, 104415 (2004).

[35] M. Zhitomirsky and A. Honecker, J. Stat. Mech.: Theor. Exp. P07012 (2004).

[36] M. Zhitomirsky and H. Tsunetsugu, Phys. Rev. B 70, 100403(R) (2004).

[37] J. Schulenburg, Spinpack homepage: http://www-e.uni-magdeburg.de/jschulen/spin/ 